\title{
Modeling and Performance Assessment of the Split-Pi Used as a Storage Converter in All the Possible DC Microgrid Scenarios. Part I: Theoretical Analysis
}

\author{
Massimiliano Luna ${ }^{1}\left(\mathbb{D}\right.$, Antonino Sferlazza ${ }^{2, *(\mathbb{D})}$, Angelo Accetta ${ }^{1}\left(\mathbb{D}\right.$, Maria Carmela Di Piazza $^{1} \mathbb{( D}_{\text {, }}$ \\ Giuseppe La Tona ${ }^{1}$ (D) and Marcello Pucci ${ }^{1}$ (D) \\ 1 Istituto di Ingegneria del Mare (INM), Consiglio Nazionale delle Ricerche (CNR), Via Ugo La Malfa 153, \\ 90146 Palermo, Italy; massimiliano.luna@cnr.it (M.L.); angelo.accetta@cnr.it (A.A.); \\ mariacarmela.dipiazza@cnr.it (M.C.D.P.); giuseppe.latona@cnr.it (G.L.T.); marcello.pucci@cnr.it (M.P.) \\ 2 Dipartimento di Ingegneria (DI), Università degli Studi di Palermo, Viale delle Scienze ed.10, \\ 90128 Palermo, Italy \\ * Correspondence: antonino.sferlazza@unipa.it
}

check for

updates

Citation: Luna, M.; Sferlazza, A.; Accetta, A.; Di Piazza, M.C.; La Tona, G.; Pucci, M. Modeling and Performance Assessment of the Split-Pi Used as a Storage Converter in All the Possible DC Microgrid Scenarios. Part I: Theoretical Analysis. Energies 2021, 14, 4902. https://doi.org/10.3390/en14 164902

Academic Editor: Mohamed Benbouzid

Received: 12 July 2021

Accepted: 7 August 2021

Published: 11 August 2021

Publisher's Note: MDPI stays neutral with regard to jurisdictional claims in published maps and institutional affiliations.

Copyright: (c) 2021 by the authors. Licensee MDPI, Basel, Switzerland. This article is an open access article distributed under the terms and conditions of the Creative Commons Attribution (CC BY) license (https:/ / creativecommons.org/licenses/by/ $4.0 /)$.

\begin{abstract}
The integration of an electrical storage system (ESS) into a DC microgrid using a bidirectional DC/DC converter provides substantial benefits but requires careful design. Among such converter topologies, the Split-pi converter presents several merits at the cost of non-isolated operation. However, the few works in the literature on the Split-pi presented only closed-loop control with a single control loop; furthermore, they neglected the reactive components' parasitic resistances and did not perform any experimental validation. This work aimed at investigating the use of the Split-pi converter as a power interface between an ESS and a DC microgrid. Five typical microgrid scenarios are presented, where each of which requires a specific state-space model and a suitable control scheme for the converter to obtain high performance. In this study, two different state-space models of the converter that consider the parasitic elements are presented, the control schemes are discussed, and criteria for designing the controllers are also given. Several simulations, as well as experimental tests on a prototype realized in the lab, were performed to validate the study. Both the simulation and experimental results will be presented in part II of this work. The proposed approach has general validity and can also be followed when other bidirectional DC/DC converter topologies are employed to interface an ESS with a DC microgrid.
\end{abstract}

Keywords: Split-pi; bidirectional converter; electrical storage system; DC microgrid; droop control; current control; feed-forward control

\section{Introduction}

Over the last few years, DC distribution in terrestrial and marine power systems has attracted a growing interest in view of the implementation of the smart microgrid paradigm due to its advantages in terms of simpler and more efficient electrical architectures. Consequently, power electronic converters that interface distributed generation units, loads, and above all, electrical storage systems (ESSs) with a common DC bus are the subject of renewed interest [1-3]. ESSs have manifold beneficial impacts on DC microgrids: they allow for improving stability and resiliency, compensate for the intermittency of renewable generation, provide ramping support to generators, and act as backup power sources. Furthermore, ESSs ensure a power buffer that can be leveraged to apply suitable energy management strategies to microgrids. In particular, energy management systems (EMSs) can be used to compute the optimal values of power flows among the microgrid devices, which allow for pursuing chosen objectives, such as a minimum electricity bill, maximum efficiency, minimum fuel consumption, or minimum greenhouse gas emissions [1,4-6].

Depending on the designer's choice, the microgrid voltage can be controlled either stiffly using a single voltage generator or in a droop scheme using one or more voltage 
generators with a predefined power-sharing ratio, usually in proportion to their power rating (grid-forming generators). When an EMS is used, the other active devices of the microgrid must be controlled as current generators based on the optimal power flows that are computed by the EMS [1,5]. Therefore, depending on the specific configuration, the converter interfacing the ESS with the microgrid must be operated as a stiff voltage generator, a non-stiff voltage generator, or a current generator.

The scientific literature provides several contributions on bidirectional DC/DC converters (BDCs). Reference [7] presented an overview of BDCs, where, besides the review of both non-isolated and isolated configurations, the most relevant control schemes and switching strategies were analyzed. In some applications, galvanic isolation between the input and output side of the converter is required; in such cases, the most frequent choice is the dual active bridge converter (DAB) due to its many advantages [7-9]. However, isolation is mandatory only when very high voltage gain is needed. Non-isolated converters are thus more attractive when the goal is to improve the efficiency, size, weight, and cost of the system [7,10]. A review of non-isolated BDCs topologies was presented in [10]. The advantages and disadvantages of the considered converters were properly highlighted. For example, some converters provide an output voltage with opposite polarity than the input, some draw discontinuous current from the battery, while others require a tapped inductor or exhibit weak regulation capability or a high switch count. Overall, Tytelmaier et al. identified the half-bridge converter (HBC) and the related interleaving variants with coupled inductors as the most promising solutions from the efficiency and robustness standpoints [10]. Furthermore, Odo compared three non-isolated BDC topologies with the classical HBC in terms of their suitability for energy storage in a DC microgrid and identified the cascaded buck-boost HBC topology as the best compromise [11].

An interesting alternative is offered by the Split-pi converter, which is a non-isolated BDC that is based on two cascaded HBCs with a common bulk capacitor instead of a common inductor. As such, it is the dual topology of the cascaded buck-boost HBC that was analyzed in $[7,10,11]$. The Split-pi was initially developed for electric vehicles and patented in 2004 [12], and it is receiving increasing attention due to its distinct advantages [13-20]. It exhibits high efficiency, like the DAB, but with a reduced switch count (eight vs. four switches, of which, only two are actively commutated). Furthermore, LC filters at both ports of the Split-pi allow for small reactive components and reduce the switching noise. On the other hand, the additional phase delay introduced by such filters can slightly complicate this converter's control with respect to simpler converters $[17,18]$. Nonetheless, the control of the Split-pi is less complicated than that of the DAB because it requires conventional duty cycle control of the pulse width modulator (PWM) instead of phase shift control. An additional advantage of the Split-pi is its suitability for multiphase systems, where a significant reduction in component size and cost can be attained. These features make it attractive when high power density is required, such as in hybrid electric vehicles, renewable energy systems, and aerospace/marine/military applications [16,17].

Only three papers in the technical literature proposed applications in which the Splitpi converter was not controlled using an open-loop [16,17,19]. The aim of [17] was to study a system in which the Split-pi interfaced a flywheel with a $12 \mathrm{~V}, 120 \mathrm{~W}$ DC bus connecting a photovoltaic generator with a passive load. However, in the related control scheme, only one control loop was active at a time (either for the output voltage or the input current) using a shared controller, and the choice was made using a rule-based approach. Singhai et al. did not focus on a specific application and presented a state-space model of the Split-pi converter and a transfer function to design a closed-loop controller for its output voltage [16]. However, they considered the converter connected to a passive load and neglected the parasitic resistances. Finally, Monteiro et al. considered a Split-pi converter with a multilevel structure and controlled current or voltage in any of the two ports in an open-loop, whereas the common DC-link voltage was controlled in a closed-loop [19]. Only a few details about the control systems were given because the study mainly focused on comparing the multilevel Split-pi topology and the interleaved topology of [20]. 
Apart from [17], all these works only presented simulation results without experimental validation. Furthermore, they all studied closed-loop control with a single control loop, neglecting the reactive components' parasitic resistances. However, when the converter is used to interface an ESS with an active load, such as a DC microgrid, it is essential to control both the ESS current and the output voltage/current of the converter, as required by the microgrid designer. Furthermore, the parasitic elements cannot be neglected because they affect the losses and the maximum gain attainable using the converter.

To cover these aspects, in this work, the use of the Split-pi converter in such an application was investigated with particular attention to its model, the design of its control system, and the assessment of the expected performance in all the possible microgrid configurations. In this first part of the work, it was shown that the Split-pi must be modeled in two different ways depending on the microgrid scenario and that control schemes involving a different number of control loops are needed. In the case of the output voltage control, a feed-forward action was also required to obtain high performance. Furthermore, it was shown that conventional PI regulators alone were not sufficient to obtain the desired performance for output current control in stiff microgrids. The two statespace models considering the parasitic elements and the transfer functions of interest were given in the study, together with criteria to design the controllers. The chosen case study was a DC microgrid that was representative of both terrestrial and marine applications. In Part II of the work, a comprehensive performance assessment is presented based on simulations and experimental tests that validate the study. Finally, the proposed approach has general validity and can also be followed when other BDC topologies are used to interface a storage system with a DC microgrid.

\section{Topology, Operation Modes, and Sizing of the Split-Pi Converter}

The schematic of a symmetrical Split-pi converter is sketched in Figure 1, including its reactive components' parasitic resistances. Such a converter can be viewed as the cascaded connection of a first HBC at port 1 , a bulk capacitor, and another HBC at port 2 . Since the two HBCs are bidirectional, the whole Split-pi converter is also a BDC. If the two HBCs have equal reactive components, the Split-pi is said to be symmetrical.

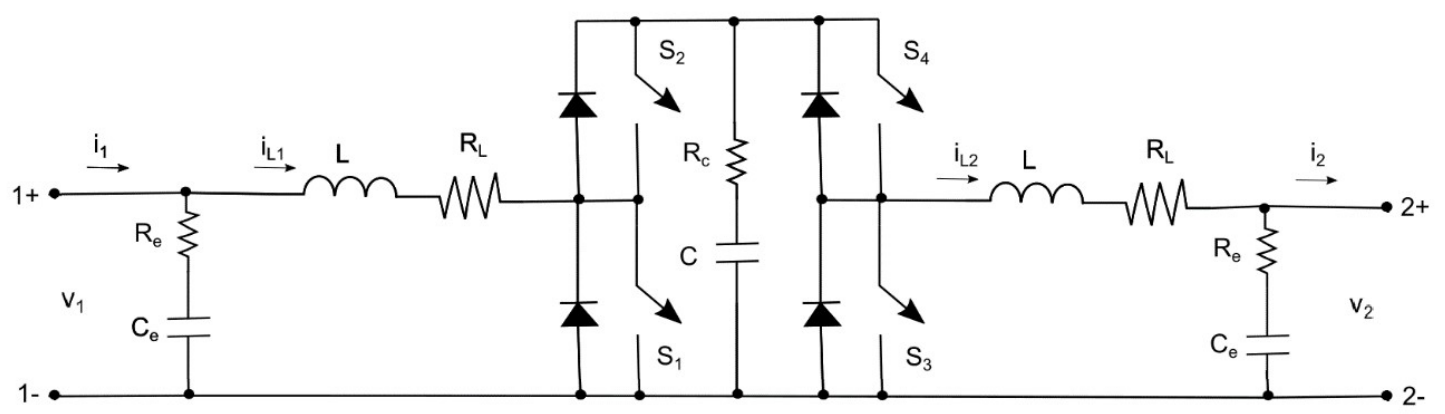

Figure 1. Schematic of the symmetrical Split-pi converter.

The four switches (S1-S4) were sketched in Figure 1 as ideal switches. In the original formulation of [12], they were implemented using MOSFETs to exploit the advantage of synchronous rectification. According to [12], the Split-pi has four operation modes, depending on the relationship between $V_{1}$ and $V_{2}$ and on the power direction, as summarized in the first four rows of Table 1, which is an extension of the table reported in [18]. In this work, the Split-pi converter was used to interface a storage system (connected to port 1) with a DC microgrid (connected to port 2). It is worth noting that, whenever possible, the storage system is chosen so that $V_{1} \leq V_{2}$ for both technical and safety reasons. Hence, the present work considered the Split-pi converter operating in modes 1 and 2. 
Table 1. Summary of the operating modes of the Split-pi converter.

\begin{tabular}{|c|c|c|c|c|c|c|c|c|}
\hline Mode & $V_{1} \leq V_{2}$ & $\begin{array}{c}\text { Power } \\
\text { Direction }\end{array}$ & $\begin{array}{c}\text { Split-Pi } \\
\text { Operation }\end{array}$ & $\begin{array}{c}\text { Duty Cycle } \\
\text { of S1 }\end{array}$ & $\begin{array}{c}\text { Duty Cycle } \\
\text { of S2 }\end{array}$ & $\begin{array}{l}\text { Duty Cycle } \\
\text { of S3 }\end{array}$ & $\begin{array}{l}\text { Duty Cycle } \\
\text { of S4 }\end{array}$ & $\begin{array}{c}\text { Gain of the } \\
\text { Split-Pi } \\
\text { in Direction } 1 \rightarrow 2\end{array}$ \\
\hline 1 & Yes & $1 \rightarrow 2$ & Boost for $1 \rightarrow 2$ & $d$ & $1-d$ & 0 & 1 & $\frac{1}{1-\lambda}$ \\
\hline 2 & Yes & $2 \rightarrow 1$ & Buck for $2 \rightarrow 1$ & $d$ & $1-d$ & 0 & 1 & $\frac{1}{1-d}$ \\
\hline 3 & No & $1 \rightarrow 2$ & Buck for $1 \rightarrow 2$ & 0 & 1 & $1-d$ & $d$ & $d$ \\
\hline 4 & No & $2 \rightarrow 1$ & Boost for $2 \rightarrow 1$ & 0 & 1 & $1-d$ & $d$ & $d$ \\
\hline
\end{tabular}

The Split-pi components can be sized according to the classical formulas used for buck and boost converters $[17,21]$. Specifically, the minimum inductance value is expressed by (1) for both boost and buck operations; on the other hand, the minimum capacitance of the input and output capacitors $C_{e}$ can be computed using (2), which is valid for a buck converter, whereas the minimum value of the bulk capacitance $C$ is expressed by (3), which is valid for a boost converter:

$$
\begin{gathered}
L_{\text {min }}=\frac{100 V_{2}(1-d) d}{2 F_{s w} r_{i \%} I_{1}}, \\
C_{e, \text { min }}=\frac{100(1-d)}{8 F_{s w}^{2} r_{v e \%} L}, \\
C_{\text {min }}=\frac{100 I_{2} d}{F_{s w} r_{v} V_{2}} .
\end{gathered}
$$

In (1), (2), and (3), $F_{s w}$ is the switching frequency; $d$ is the duty cycle; $V_{2}$ and $I_{2}$ are the output voltage and current, respectively; $I_{1}$ is the input current; $r_{i} \%$ is the desired inductor current ripple; and $r_{v \%}$ and $r_{v e \%}$ are the desired voltage ripple values on the bulk and external capacitors, respectively.

\section{Possible DC Microgrid Scenarios, Load Models, and Required Control Schemes for the ESS Converter}

In the present study, it was supposed that the Split-pi converter was used to interface an ESS with a DC microgrid. Under this hypothesis, several scenarios could be considered depending on the control mode chosen for both the storage-side converter and the grid-side converters interfacing other microgrid generators, if any. Depending on the combinations of the control modes of such converters, five scenarios were identified and, for each of them, a different type of load model and control scheme must be used. The different DC microgrid scenarios and the required load models and control schemes are discussed in the following subsections.

\subsection{Possible DC Microgrid Scenarios}

In general terms, one of the following situations can occur as a control mode for the microgrid converters:

- All the generator converters are controlled in droop mode to regulate the microgrid voltage with a predefined power-sharing ratio. Thus, each generator behaves as an ideal voltage generator with a series-connected resistance. The droop scheme leads to a simple but very reliable microgrid and does not require a communication infrastructure. If a storage system is present, its droop characteristic is usually chosen so that no current is supplied at half the rated load of the microgrid, whereas charging/discharging occurs for load power below/above such a value. If there is only one active device in the microgrid (either a storage system or a generator), it could also be controlled with a null droop resistance (stiff microgrid) so that the microgrid voltage does not vary with load power

- The microgrid follows a master-slave architecture: some converters for generators or storage systems (i.e., the masters) are controlled in droop mode (a null droop resistance 
is possible only with a single master); the others (i.e., the slaves) behave as current generators and are managed by an EMS to pursue one or more predefined goals.

Therefore, three control modes are possible for both the storage-side and grid-side converters: stiff droop control, non-stiff droop control, and current control. The five scenarios resulting from the combinations of the control modes of such converters are described in the first three columns of Table 2. For the sake of clarity, such scenarios are referred to also by means of an abbreviation in the form Sx-Gy, where $\mathrm{x}$ and $\mathrm{y}$ specify the control mode for the storage-side and the grid-side converters, respectively. The range of options for $\mathrm{x}$ is:

- C for current control.

- D for non-stiff droop control.

- $\quad$ S for stiff droop control.

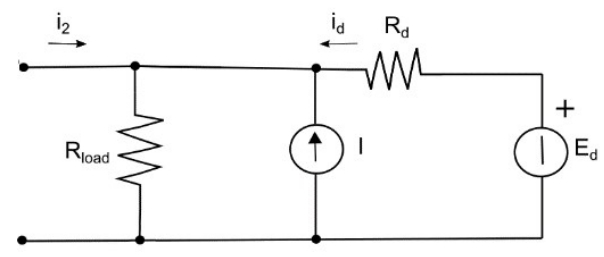

(a)

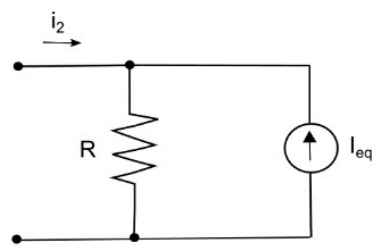

(b)

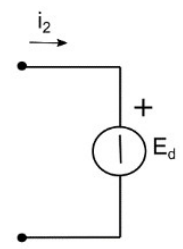

(c)

Figure 2. Equivalent active load transformations for non-stiff and stiff microgrids: (a) general load model for storage converter; (b) equivalent load model considered in scenarios \#1-\#4; (c) equivalent load model considered in scenario \#5.

Table 2. Possible combinations of microgrid scenarios, converter and load models, and control schemes for the Split-pi.

\begin{tabular}{|c|c|c|c|c|c|}
\hline Microgrid Scenario & $\begin{array}{l}\text { Storage } \\
\text { Converter }\end{array}$ & Other Generators & $\begin{array}{l}\text { State-Space Model of } \\
\text { the Split-Pi }\end{array}$ & $\begin{array}{c}\text { Load for the } \\
\text { State-Space Model of } \\
\text { the Split-Pi }\end{array}$ & Control Scheme \\
\hline $\begin{array}{c}\# 1 \\
\text { (SS-GN) }\end{array}$ & $\begin{array}{c}\text { Droop mode with } \\
R_{d}=0 \text { (stiff microgrid) }\end{array}$ & $\begin{array}{l}\text { No other generator } \\
\text { present (passive load) or } \\
\text { all current controlled by } \\
\text { the EMS }\end{array}$ & Model A & $\begin{array}{c}R \text { and } I_{e q} \\
\text { as in Figure } 2 b\end{array}$ & $\begin{array}{c}2 \text { loops }+\mathrm{FF} \text {, as in } \\
\text { Figure } 3 \text { with } R_{d s}=0\end{array}$ \\
\hline $\begin{array}{c}\# 2 \\
\text { (SD-GN) }\end{array}$ & $\begin{array}{l}\text { Droop mode with } \\
\qquad R_{d} \neq 0\end{array}$ & $\begin{array}{l}\text { No other generator } \\
\text { present (passive load) or } \\
\text { all current controlled by } \\
\text { the EMS }\end{array}$ & Model A & $\begin{array}{c}R \text { and } I_{e q} \\
\text { as in Figure } 2 b\end{array}$ & $\begin{array}{l}3 \text { loops + FF, as } \\
\text { in Figure } 3\end{array}$ \\
\hline $\begin{array}{c}\# 3 \\
(\mathrm{SD}-\mathrm{GD})\end{array}$ & $\begin{array}{l}\text { Droop mode with } \\
\qquad R_{d} \neq 0\end{array}$ & $\begin{array}{l}\text { At least one is droop } \\
\text { controlled and none has } \\
R_{d}=0\end{array}$ & Model A & $\begin{array}{l}R \text { (low) and } I_{e q}, \\
\text { as in Figure } 2 \mathrm{~b}\end{array}$ & $\begin{array}{l}3 \text { loops }+ \text { FF, as } \\
\text { in Figure } 3\end{array}$ \\
\hline $\begin{array}{c}\# 4 \\
(\mathrm{SC}-\mathrm{GD})\end{array}$ & Current mode & $\begin{array}{l}\text { At least one is droop } \\
\text { controlled and none has } \\
R_{d}=0\end{array}$ & Model A & $\begin{array}{l}R \text { (low) and } I_{e q}, \\
\text { as in Figure } 2 \mathrm{~b}\end{array}$ & 2 loops, as in Figure 4 \\
\hline $\begin{array}{c}\# 5 \\
\text { (SC-GS) }\end{array}$ & Current mode & $\begin{array}{l}\text { One is droop controlled } \\
\text { and has } R_{d}=0 \text { (stiff } \\
\text { microgrid); the others, if } \\
\text { present, are current } \\
\text { controlled by the EMS }\end{array}$ & Model B & $\begin{array}{c}E_{d \prime} \\
\text { as in Figure 2c }\end{array}$ & 2 loops, as in Figure 4 \\
\hline
\end{tabular}

As for the options for $\mathrm{y}$ :

- $\mathrm{N}$ if no grid-side generator is present or if none of the grid-side generators are operated with droop control.

- D if at least one generator is droop controlled but not in a stiff way.

- $\quad \mathrm{S}$ if there is one generator operated with stiff droop control; in such a case, the other grid-side generators, if present, must be current controlled by the EMS.

\subsection{Possible Load Models for the ESS Converter}

The dynamic behavior of the storage converter is affected by the load that it must supply, which depends not only on the passive loads of the DC microgrid but also on the possible presence of grid-side generators. In the most general case, the load for the ESS 
converter can be modeled following these steps: (1) aggregating all the droop-controlled generators of the microgrid using Thevenin's theorem, obtaining parameters $E_{d}$ and $R_{d}$; (2) combining all the current-controlled generators managed by the EMS to compute the overall current $I$; (3) including an aggregated passive load $R_{\text {load }}$. The resulting circuit model is shown in Figure 2a. However, it is convenient to reduce such a model to a suitable equivalent form comprising only one generator. Toward this aim, it is necessary to distinguish between the five scenarios. In scenarios \#3 (SD-GD) and \#4 (SC-GD), using Norton's theorem, the couple $E_{d}, R_{d}$ can be substituted with an equivalent current generator $E_{d} / R_{d}$ and a parallel-connected resistance $R_{d}$. Then, the load can be reduced to that of Figure $2 \mathrm{~b}$ with the following assumptions: $I_{e q}=I+E_{d} / R_{d}$ and $R=R_{\text {load }} / / R_{d}$, where // denotes the parallel connection of circuit elements. In scenarios \#1 (SS-GN) and \#2 (SD-GN), the same scheme of Figure $2 \mathrm{~b}$ can be used assuming $I_{e q}=I$ and $R=R_{\text {load }}$. Furthermore, in scenario \#5 (SC-GS), the converter's output voltage is set equal to $E_{d}$ because $R_{d}=0$. Since both $R_{\text {load }}$ and $I$ are now parallel connected to an ideal voltage generator, they do not influence the converter's dynamics. Thus, the load can be modeled as shown in Figure 2c.

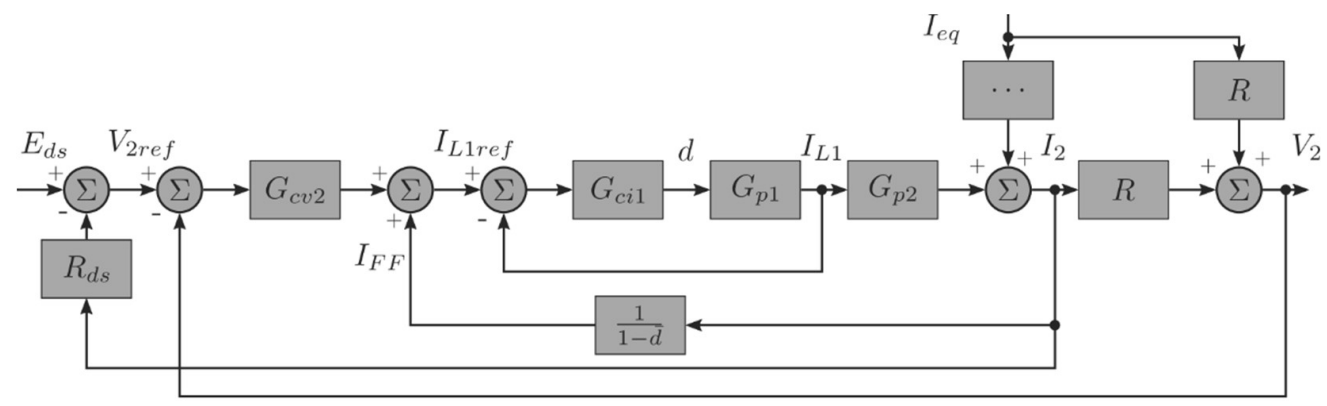

Figure 3. Control scheme that is used for the voltage control of the ESS converter in scenarios \#1 (SS-GN), \#2 (SD-GN), and \#3 (SD-GD).

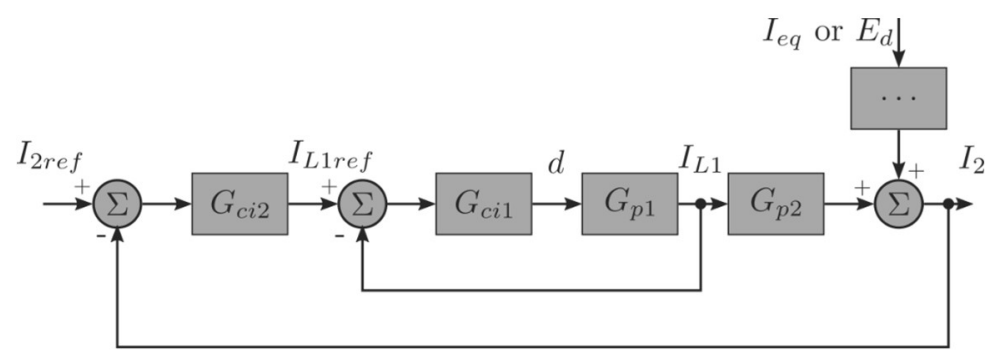

Figure 4. Control scheme that is used for the current control of the ESS converter in scenarios \#4 (SC-GD) and \#5 (SC-GS).

In the following, the state-space model of the storage converter connected to the load of Figure $2 \mathrm{~b}$ is denoted as model $\mathrm{A}$, whereas model $\mathrm{B}$ refers to the converter connected to the load of Figure 2c. The derivation of such models is given in Section 4. The relationship between the possible DC microgrid scenarios and the storage converter and load models is described in the first five columns of Table 2. It is worth noting that the value of the equivalent load resistance $R$ considered in scenarios \#3 (SD-GD) and \#4 (SC-GD) is much lower than that of scenarios \#1 (SS-GN) and \#2 (SD-GN) because it results from the parallel connection of $R_{\text {load }}$ and $R_{d}$. Finally, the last column of Table 2 reports the required control scheme for the ESS converter in each microgrid scenario according to the considerations given in the following section.

\subsection{Required Control Schemes for the ESS Converter}

In general, the closed-loop control scheme for the storage converter depends on the microgrid scenario. However, regardless of the scenario, an important goal is to control the current $I_{L 1}$ of the leftmost inductor to ensure that it is compatible with the storage system's 
current state. Thus, in the related control loop, its reference value should be dynamically saturated to avoid overcharging or overdischarging the storage system.

In the first three scenarios, i.e., SS-GN, SD-GN, and SD-GD, besides the current loop for $I_{L 1}$, a voltage loop is needed to regulate the output voltage $V_{2}$ at the nominal voltage $V_{2 n}$. A suitable controller is required in each control loop. Typically, PI or PID regulators with an anti-windup action are used, which must be designed to obtain a stable system with the desired dynamics. In scenarios \#2 (SD-GN) and \#3 (SD-GD), a third loop is also required to implement the droop characteristic of the storage converter by computing the voltage reference $V_{2 \text { ref }}$ based on the output current $I_{2}$ according to the equation $V_{2, r e f}=E_{d s}-R_{d s} \cdot I_{2}$, where $E_{d s}$ and $R_{d s}$ are the parameters of the droop characteristic of such a converter. The control scheme used in scenarios \#1 (SS-GN), \#2 (SD-GN), and \#3 (SD-GD) is depicted in Figure 3. In particular, the external loop is opened in scenario \#1 (SS-GN) because $R_{d}=0$. The presence of the current generator $I_{e q}$ in parallel to the load resistance is considered a disturbance that affects the system's output, which will be suitably compensated for by the control system, regardless of the related transfer function. As will be shown in Section 6, when the output voltage $V_{2}$ is controlled, a feed-forward (FF) action is also required in addition to the voltage loop to suitably reduce the overshoot. Since $I_{e q}$ cannot be measured, the output current $I_{2}$ is chosen as the FF action input.

On the other hand, the converter is current-controlled in scenarios \#4 (SC-GD) and \#5 (SC-GS). In these cases, besides the inner loop for $I_{L 1}$, another current loop is needed to regulate the output current $I_{2}$ based on a reference $I_{2 r e f}$ that is computed by the EMS. The related control scheme is shown in Figure 4. Again, the external voltage or current generator is considered a disturbance, and the related transfer function is irrelevant.

The type of control scheme to be used in each microgrid scenario and the required number of control loops are reported in the last column of Table 2. It is worth noting that suitable saturators are required in the controllers of each loop $\left(G_{c i 1}, G_{c v 2}, G_{c i 2}\right)$. Specifically, the output of the controller $G_{c i 1}$ (i.e., the duty cycle $d$ ) is bounded by the interval [0; 0.9] to avoid overcurrents due to prolonged transients with $d=1$. On the other hand, the reference value for $I_{L 1}$ (i.e., the output of $G_{\mathcal{C}}$ plus the FF term or the output of $G_{c i 2}$ ) is bounded by the interval $\left[-I_{c x} ; I_{d x}\right]$, where $I_{c x}$ and $I_{d x}$ are the maximum charging/discharging currents of the storage system. Finally, the upper or lower bound of such an interval is dynamically replaced with zero if the battery SOC reaches $100 \%$ or goes below the minimum allowed SOC, respectively.

\section{State-Space Models of the Split-Pi Converter}

The two state-space models of a Split-pi converter that interfaces a storage system with a non-stiff (scenarios \#1 \#4) or stiff (scenario \#5) microgrid and operates with $V_{1} \leq V_{2}$ are presented in the following. They consider the parasitic elements and were determined according to the state-space averaging technique [22].

\subsection{State-Space Model A: Split-Pi Converter Connected to a Non-Stiff Microgrid}

The state-space model of a Split-pi converter connected to a non-stiff microgrid and operating with $V_{1} \leq V_{2}$ can be expressed in matrix form as follows:

$$
\begin{gathered}
\left\{\begin{array}{l}
\dot{x}=A x+B u \\
y=C x+D u
\end{array}\right. \\
x=\left[I_{L 1}, I_{L 2}, V_{c}, V_{e}\right]^{\prime} \\
u=\left[V_{1}, I_{e q}\right]^{\prime} \\
y=\left[I_{L 1}, V_{2}, I_{2}\right]^{\prime}
\end{gathered}
$$




$$
\begin{aligned}
& \left\{\begin{array}{c}
A=d A_{o n}+(1-d) A_{o f f} \\
B=d B_{o n}+(1-d) B_{o f f} \\
C=d A_{o n}+(1-d) C_{o f f} \\
D=d D_{o n}+(1-d) D_{o f f}
\end{array}\right. \\
& A_{\text {on }}=\left[\begin{array}{cccc}
-\frac{R_{L}}{L} & 0 & 0 & 0 \\
0 & -\frac{R_{\text {tot }}}{L} & \frac{1}{L} & -\frac{R}{L R_{\text {sum }}} \\
0 & -\frac{1}{C} & 0 & 0 \\
0 & \frac{R}{R_{\text {sum }} C_{e}} & 0 & -\frac{1}{R_{\text {sum }} C_{e}}
\end{array}\right] \\
& A_{o f f}=\left[\begin{array}{cccc}
-\frac{R_{L}+R_{c}}{L} & \frac{R_{c}}{L} & -\frac{1}{L} & 0 \\
\frac{R_{c}}{L} & -\frac{R_{\text {tot }}}{L} & \frac{1}{L} & -\frac{R}{L R_{\text {sum }}} \\
\frac{1}{C} & -\frac{1}{C} & 0 & 0 \\
0 & \frac{R}{R_{\text {sum }} C_{e}} & 0 & -\frac{1}{R_{\text {sum }} C_{e}}
\end{array}\right] \\
& B_{o n}=B_{o f f}=\left[\begin{array}{cc}
\frac{1}{L} & 0 \\
0 & -\frac{R_{p}}{L} \\
0 & 0 \\
0 & \frac{R}{R_{s u m} C_{e}}
\end{array}\right] \\
& C_{o n}=C_{o f f}=\left[\begin{array}{cccc}
1 & 0 & 0 & 0 \\
0 & R_{p} & 0 & \frac{R}{R_{\text {sum }}} \\
0 & \frac{R_{e}}{R_{\text {sum }}} & 0 & \frac{1}{R_{\text {sum }}}
\end{array}\right] \\
& D_{\text {on }}=D_{\text {off }}=\left[\begin{array}{cc}
0 & 0 \\
0 & R_{p} \\
0 & -\frac{R}{R_{\text {sum }}}
\end{array}\right]
\end{aligned}
$$

where $R_{p}=R / / R_{e}, R_{\text {sum }}=R+R_{e}$, and $R_{t o t}=R_{p}+R_{L}+R_{c}$.

Since $B_{o n}=B_{\text {off } f}$ and $D_{o n}=D_{o f f}$, the small-signal behavior of the converter does not depend on the input values. According to the method described in [21], the model can be linearized around a chosen operating point corresponding to the duty cycle $\bar{d}$ and the state $\bar{x}=\left[I_{L 10}, I_{L 20}, V_{c 0}, V_{e 0}\right]^{\prime}$, obtaining the following transfer functions:

$$
\begin{gathered}
G_{p 1}(s)=\frac{\widetilde{I}_{L 1}}{\widetilde{d}}=\frac{n_{3} s^{3}+n_{2} s^{2}+n_{1} s+n_{0}}{d_{4} s^{4}+d_{3} s^{3}+d_{2} s^{2}+d_{1} s+d_{0}} \\
G_{p 2}(s)=\frac{\widetilde{I}_{2}}{\widetilde{I}_{L 1}}=\frac{R_{k} I_{L 10}}{L^{2} C C_{e} R_{s u m}} \cdot \frac{\left(1+s R_{c} C\right)\left(1+s R_{e} C_{e}\right)\left(1-s \frac{L}{R_{k}}\right)}{n_{3} s^{3}+n_{2} s^{2}+n_{1} s+n_{0}}
\end{gathered}
$$

whose coefficients $n_{i}, d_{i}$, and $R_{k}$ are given in Appendix A. $G_{p 1}(s)$ and $G_{p 2}(s)$ are small-signal transfer functions that express the effect of perturbations of a variable on another one (both denoted with a tilde). According to Figure $3, G_{p 1}(s)$ expresses the relationship between $d$ and $I_{L 1}$, whereas $G_{p 2}(s)$ describes the dependence of $I_{2}$ on $I_{L 1}$. Clearly, the relationship between $I_{L 1}$ and $V_{2}$ is expressed by $G_{p 2}(s) \cdot R$.

\subsection{State-Space Model B: Split-Pi Converter Connected to a Stiff Microgrid}

In the case of a Split-pi converter operating with $V_{1} \leq V_{2}$ and connected to a stiff microgrid, (4) and (8) are still valid, but (5), (6), (7),(9), (10) and (11), (12), (13) are replaced by (16), (17), (18), (19), (20) and (21), (22), (23), respectively.

$$
\begin{gathered}
x=\left[I_{L 1}, I_{L 2}, V_{c}, V_{e}\right]^{\prime} \\
u=\left[V_{1}, E_{d}\right]^{\prime} \\
y=\left[I_{L 1}, I_{2}\right]^{\prime}
\end{gathered}
$$




$$
\begin{aligned}
& A_{o n}=\left[\begin{array}{cccc}
-\frac{R_{L}}{L} & 0 & 0 & 0 \\
0 & -\frac{R_{c}+R_{L}}{L} & \frac{1}{L} & 0 \\
0 & -\frac{1}{C} & 0 & 0 \\
0 & 0 & 0 & -\frac{1}{R_{e} C_{e}}
\end{array}\right] \\
& A_{o f f}=\left[\begin{array}{cccc}
-\frac{R_{c}+R_{L}}{L} & \frac{R_{c}}{L} & -\frac{1}{L} & 0 \\
\frac{R_{c}}{L} & -\frac{R_{c}+R_{L}}{L} & \frac{1}{L} & 0 \\
\frac{1}{C} & -\frac{1}{C} & 0 & 0 \\
0 & 0 & 0 & -\frac{1}{R_{e} C_{e}}
\end{array}\right] \\
& B_{o n}=B_{o f f}=\left[\begin{array}{cc}
\frac{1}{L} & 0 \\
0 & -\frac{1}{L} \\
0 & 0 \\
0 & \frac{1}{R_{e} C_{e}}
\end{array}\right] \\
& C_{o n}=C_{o f f}=\left[\begin{array}{cccc}
1 & 0 & 0 & 0 \\
0 & 1 & 0 & \frac{1}{R_{e}}
\end{array}\right] \\
& D_{o n}=D_{o f f}=\left[\begin{array}{cc}
0 & 0 \\
0 & -\frac{1}{R_{e}}
\end{array}\right]
\end{aligned}
$$

Again, the small-signal behavior does not depend on input values. The model can be linearized around a chosen operating point corresponding to $\bar{d}$ and $\bar{x}$, obtaining the transfer functions (24) and (25), whose coefficients $n_{i}, d_{i}$, and $R_{k}$ are given in Appendix B. According to Figure $4, G_{p 1}(s)$ expresses the relationship between $d$ and $I_{L 1}$, whereas $G_{p 2}(s)$ describes the dependence of $I_{2}$ on $I_{L 1}$. It is worth noting that a zero-pole cancellation occurs due to the stiff voltage imposed on the $R_{e} C_{e}$ branch at port 2; thus, the resulting system's order is three instead of four and the voltage and current on $C_{e}$ cannot be controlled.

$$
\begin{gathered}
G_{p 1}(s)=\frac{\widetilde{I}_{L 1}}{\widetilde{d}}=\frac{n_{2} s^{2}+n_{1} s+n_{0}}{d_{3} s^{3}+d_{2} s^{2}+d_{1} s+d_{0}} \\
G_{p 2}(s)=\frac{\widetilde{I}_{2}}{\widetilde{I}_{L 1}}=\frac{R_{k} I_{L 10}}{L^{2} C} \cdot \frac{\left(1+s R_{c} C\right)\left(1-s \frac{L}{R_{k}}\right)}{n_{2} s^{2}+n_{1} s+n_{0}}
\end{gathered}
$$

\section{Case Study and Converter Sizing}

In this section, the chosen case study is described and the droop characteristics of both the storage converter and the voltage generator of the microgrid are discussed. Then, the Split-pi converter's reactive components are sized based on the chosen case study's parameters.

With no loss of generality, the proposed investigation was performed by referring to a $48 \mathrm{~V}, 750 \mathrm{~W}$ storage system that was interfaced with a $180 \mathrm{~V}$ DC microgrid using a Split-pi converter. The chosen case study can represent the onboard grid of an unmanned marine vehicle or a scaled prototype of a residential DC microgrid with a $120 \mathrm{~V}, 60 \mathrm{~Hz}$, single-phase, grid-connected inverter, whose DC link voltage must be higher than $170 \mathrm{~V}$ DC. The rated values of the system under study are shown in Table 3.

As for the chosen droop characteristic of the storage converter, in scenario \#1 (SS-GN) it was defined by $E_{d s}=180 \mathrm{~V}$ and $R_{d s}=0$, whereas in scenarios \#2 (SD-GN) and \#3 (SD-GD), it was expressed by $E_{d s}=180 \mathrm{~V}$ and $R_{d s}=2.2 \Omega$, i.e., imposing a $5 \%$ voltage reduction at the nominal current. For the equivalent droop-controlled microgrid generator, the same parameters as those of the storage converter were chosen in scenario \#4 (SC-GD). On the other hand, in scenario \#3 (SD-GD), the following parameters were used for the droopcontrolled microgrid generator: $E_{d}=198 \mathrm{~V}$ and $R_{d}=9 \Omega$; with this choice, the storage system did not supply any power for half the rated load of the microgrid, as desired. 
Finally, a constant voltage generator $E_{d}=180 \mathrm{~V}$ was considered in scenario \#5 (SC-GS) to model the microgrid's stiff voltage generator.

Table 3. Rated values of the system.

\begin{tabular}{ccc}
\hline Parameter & Symbol & Value \\
\hline Switching frequency & $F_{s w}$ & $20 \mathrm{kHz}$ \\
Nominal output voltage & $V_{2 n}$ & $180 \mathrm{~V}$ \\
Nominal input voltage & $V_{1 n}$ & $50 \mathrm{~V}$ \\
Nominal power & $P_{n}$ & $750 \mathrm{~W}$ \\
Nominal load resistance & $R_{n}$ & $43.2 \Omega$ \\
Nominal input current & $I_{1 n}$ & $15 \mathrm{~A}$ \\
Max. charge/discharge current & $I_{c x}, I_{d x}$ & $18 \mathrm{~A}$ \\
Nominal output current & $I_{2 n}$ & $4.167 \mathrm{~A}$ \\
Nominal duty cycle & $d$ & 0.722 \\
\hline
\end{tabular}

The Split-pi's reactive components were sized using (1), (2), (3), and the following parameters were set: $r_{i \%}= \pm 6.0 \%, r_{v e} \%= \pm 0.2 \%, r_{v} \%= \pm 0.2 \%$. Consequently, the following minimum inductor and capacitor ratings were obtained: $L_{\min }=803 \mu \mathrm{H}, C_{e, \min }=113 \mu \mathrm{F}$, $C_{\min }=419 \mu \mathrm{F}$. Aiming to build a converter prototype and due to component availability, slightly higher values were chosen for the reactive components; they are reported in Table 4, together with their parasitic resistances.

Table 4. Reactive components of the Split-pi.

\begin{tabular}{ccc}
\hline Parameter & Symbol & Value \\
\hline Inductance value of $L$ & $L$ & $1000 \mu \mathrm{H}$ \\
Parasitic resistance of $L$ & $R_{L}$ & $65 \mathrm{~m} \Omega$ \\
Capacitance value of $C_{e}$ & $C_{e}$ & $200 \mu \mathrm{F}$ \\
Parasitic resistance of $C_{e}$ & $R_{e}$ & $260 \mathrm{~m} \Omega$ \\
Capacitance value of $C$ & $C$ & $540 \mu \mathrm{F}$ \\
Parasitic resistance of $C$ & $R_{c}$ & $125 \mathrm{~m} \Omega$ \\
\hline
\end{tabular}

\section{Control System Design}

The control system of the Split-pi converter must be designed by considering the specific microgrid scenario in which it will be used. However, in any case, it must be assumed that the converter supplies its rated power without any contribution from the external current or voltage generators, which are seen as disturbances. If the imposed stability margins are sufficiently wide, the designed controllers will be effective also at lighter loads, i.e., with higher values of $R$. Thus, models $\mathrm{A}$ and $\mathrm{B}$ must be linearized around the operating point corresponding to the rated values of the duty cycle and state variables: $\bar{d}=0.722$ and $\bar{x}=\left[I_{L 10}, I_{L 20}, V_{c 0}, V_{e 0}\right]^{\prime}=[15,4.167,180,180]^{\prime}$. No other condition is needed for model B in scenario \#5 (SC-GS). As for model A, instead, it is $R=R_{n}$ in scenarios $\# 1$ (SS-GN) and \#2 (SD-GN) and $R=R_{n} / / R_{d}$ in scenarios \#3 (SD-GD) and \#4 (SC-GD).

All the above values must be substituted into (A1)-(A6), given in Appendix A, to obtain the coefficients of the transfer functions of interest. Then, designing of the controllers $G_{c i 1}, G_{c v 2}$, and $G_{c i 2}$ can be performed with classic techniques that involve imposing suitable values of the crossover frequency $\omega_{c}$ and phase margin $\mathrm{m}_{\varphi}$ and ensuring a suitable gain margin $\mathrm{m}_{\mathrm{g}}$ [23]. For each control loop and scenario, the imposed values and the obtained PI coefficients and gain margins are summarized in Tables 5 and 6. Furthermore, a baseline scenario employing the control scheme of Figure 3 with $R_{d}=0$ and without the FF action was also considered for comparison purposes to show the usefulness of such an action. 
Table 5. Coefficients of PI regulators in the case of model A.

\begin{tabular}{|c|c|c|c|c|c|c|}
\hline Loop & Scenario & Value of $R$ & Controller & Imposed $\omega_{c}$ and $m_{\varphi}$ & PI Coefficients & Obtained $\mathrm{m}_{\mathrm{g}}$ \\
\hline Current $I_{L 1}$ & $\begin{array}{c}\text { Baseline } \\
\# 1 \text { (SS-GN) } \\
\# 2 \text { (SD-GN) }\end{array}$ & $R_{n}$ & $G_{c i_{1}}$ & $\omega_{\mathrm{c}}=3000 \mathrm{rad} / \mathrm{s}$ and $\mathrm{m}_{\varphi}=85^{\circ}$ & $\begin{array}{l}K_{p i}=0.0160 \\
K_{i i}=5.3703\end{array}$ & $\infty$ \\
\hline Current $I_{L 1}$ & $\begin{array}{l}\# 3 \text { (SD-GD) } \\
\# 4 \text { (SC-GD) }\end{array}$ & $R_{n} / / R_{d}$ & $G_{c i_{1}}$ & $\omega_{\mathrm{c}}=3000 \mathrm{rad} / \mathrm{s}$ and $\mathrm{m}_{\varphi}=85^{\circ}$ & $\begin{array}{l}K_{p i}=0.0161 \\
K_{i i}=5.2481\end{array}$ & $\infty$ \\
\hline $\begin{array}{l}\text { Voltage } V_{2} \\
\text { without FF }\end{array}$ & Baseline & $R_{n}$ & $G_{\mathrm{Cv}_{2}}$ & $\omega_{\mathrm{c}}=100 \mathrm{rad} / \mathrm{s}$ and $\mathrm{m}_{\varphi}=85^{\circ}$ & $\begin{array}{r}K_{p v}=0.2659 \\
K_{i v}=18.6209\end{array}$ & $12.7 \mathrm{~dB}$ \\
\hline $\begin{array}{l}\text { Voltage } V_{2} \\
\text { with FF }\end{array}$ & $\begin{array}{l}\# 1 \text { (SS-GN) } \\
\# 2 \text { (SD-GN) }\end{array}$ & $R_{n}$ & $G_{c v_{2}}$ & $\omega_{\mathrm{c}}=100 \mathrm{rad} / \mathrm{s}$ and $\mathrm{m}_{\varphi}=85^{\circ}$ & $\begin{array}{c}K_{p v}=0.2712 \\
K_{i v}=10.4112\end{array}$ & $13.1 \mathrm{~dB}$ \\
\hline $\begin{array}{l}\text { Voltage } V_{2} \\
\text { with FF }\end{array}$ & \#3 (SD-GD) & $R_{n} / / R_{d}$ & $G_{c v_{2}}$ & $\omega_{\mathrm{c}}=100 \mathrm{rad} / \mathrm{s}$ and $\mathrm{m}_{\varphi}=85^{\circ}$ & $\begin{array}{c}K_{p v}=0.3284 \\
K_{i v}=14.6218\end{array}$ & $24.2 \mathrm{~dB}$ \\
\hline Current $I_{2}$ & \#4 (SC-GD) & $R_{n} / / R_{d}$ & $G_{c i_{2}}$ & $\omega_{\mathrm{c}}=100 \mathrm{rad} / \mathrm{s}$ and $\mathrm{m}_{\varphi}=85^{\circ}$ & $\begin{array}{c}K_{p i 2}=0.3746 \\
K_{i i 2}=389.045\end{array}$ & $23.7 \mathrm{~dB}$ \\
\hline
\end{tabular}

Table 6. Coefficients of PI regulators in the case of model B.

\begin{tabular}{|c|c|c|c|c|c|}
\hline Loop & Scenario & Controller & Imposed $\omega_{c}$ and $m_{\varphi}$ & PI Coefficients & Obtained $\mathrm{m}_{\mathrm{g}}$ \\
\hline Current $I_{L 1}$ & \#5 (SC-GS) & $G_{c i_{1}}$ & $\omega_{\mathrm{c}}=3000 \mathrm{rad} / \mathrm{s}$ and $\mathrm{m}_{\varphi}=85^{\circ}$ & $\begin{array}{l}K_{p i}=0.0161 \\
K_{i i}=5.0699\end{array}$ & $\infty$ \\
\hline Current $I_{2}$ & \#5 (SC-GS) & $G_{c i_{2}}$ & $\omega_{\mathrm{c}}=100 \mathrm{rad} / \mathrm{s}$ and $\mathrm{m}_{\varphi}=85^{\circ}$ & $\begin{array}{l}K_{p i 2}=1.1953 \\
K_{i i 2}=362.22\end{array}$ & $\begin{array}{c}13.6 \mathrm{~dB} \\
\text { with } G_{a d d}(s)\end{array}$ \\
\hline
\end{tabular}

It is worth noting that the design of the controllers must be very conservative. By design, the currents $I_{L 1}$ and $I_{L 2}$ have a significant switching ripple compared to the input/output currents and voltages. Thus, the desired crossover frequency for the $I_{L 1}$ loop must be suitably lower than $F_{s w}$ to avoid the switching ripple being processed by the controller. The crossover frequency of the loop for $V_{2}$ or $I_{2}$ must be even lower for proper decoupling with respect to the inner loop. When the converter supplies a passive load $\left(R_{\text {load }}\right)$, some ringing can be tolerated, and the usually adopted phase margin $\left(50-60^{\circ}\right)$ is satisfactory. Instead, in the case of an active microgrid, the combined variations of $I$ and $R_{\text {load }}$ could determine a significant excursion from the nominal operating point and pronounced under/overshoots; thus, a higher phase margin (i.e., $\mathrm{m}_{\varphi}>80^{\circ}$ ) is required to ensure stability under all the operating conditions. As for the gain margin, the usually adopted criterion (i.e., $\mathrm{m}_{\mathrm{g}}>12 \mathrm{~dB}$ ) is enough to ensure robustness against parameter variations.

As for the chosen case study, some noteworthy remarks can be made:

- Regardless of the value of $R$, models $\mathrm{A}$ and $\mathrm{B}$ exhibited nearly the same dynamics for $I_{L 1}$; therefore, the corresponding PI regulators $G_{c i 1}$ had similar coefficients. On the other hand, the dynamics of $I_{2}$ were quite dissimilar and required different $G_{c i 2}$ controllers.

- In the case of model $B$, it could be possible to achieve the desired $\omega_{c}$ and $m_{\varphi}$ with a PI or I regulator alone, but the gain margin would be around $6.6 \mathrm{~dB}$ due to a resonance peak; thus, the second-order transfer function (26) must be included after the PI regulator to attenuate such a peak and achieve a gain margin of $13.6 \mathrm{~dB}$.

- The dynamics of $I_{2}$ were very sensitive to the value of $R$ in model $\mathrm{A}$; thus, an unstable system was obtained in scenario \#4 (SC-GD) if the controllers were designed assuming $R=R_{n}$ instead of $R=R_{n} / / R_{d}$.

- Without the FF action, the dynamics of $V_{2}$ were sensitive to the value of $R$ : a significantly slower behavior was obtained if the controllers were designed assuming $R=R_{n}$ in scenario \#3 (SD-GD) instead of $R=R_{n} / / R_{d}$; on the other hand, a significant ringing was obtained if the controllers were designed considering $R=R_{n} / / R_{d}$ in scenarios $\# 1$ (SS-GN) and \#2 (SD-GN) instead of $R=R_{n}$. 
- Using the FF action, the dynamics of $V_{2}$ were pretty insensitive to the value of $R$; thus,

almost no variation was obtained in scenarios \#1 (SS-GN), \#2 (SD-GN), and \#3 (SD-GD) if the controllers were designed when considering either $R=R_{n} / / R_{d}$ or $R=R_{n}$.

Several simulations were performed to assess the performance of the controlled system in all the scenarios. Then, a prototype of the Split-pi converter was built, and experimental tests were performed in several conditions that covered the baseline scenario and all the other five scenarios, obtaining successful results. The simulation and experimental results validating the study are presented in part II of this work.

$$
G_{a d d}(s)=\frac{1}{\left(1+\frac{s}{533}\right)\left(1+\frac{s}{606}\right)}
$$

\section{Conclusions}

The Split-pi converter is a suitable choice to interface electrical storage systems with DC microgrids. It offers distinct advantages, such as high efficiency, reduced switch count and switching noise, and suitability for multiphase systems at the cost of non-isolated operation. However, to obtain high performance, its control system must be suitably designed according to the specific microgrid scenario in which it will be used.

In this study, five typical microgrid scenarios were identified and analyzed, where each of which required a specific state-space model and a suitable control scheme for the converter. Two different state-space models were presented for the Split-pi converter operating with the storage-side voltage being lower than the grid-side voltage. Both models considered the parasitic elements of the reactive components. As for the control scheme, the number of required control loops depended on the scenario. It was shown that feed-forward action is needed to obtain a high performance in the case of voltage control and that, sometimes, conventional PI regulators alone were not sufficient for stable current control. The most relevant transfer functions of the Split-pi converter were given, together with criteria to design the controllers. Several simulations, as well as experimental tests on a prototype realized in the lab, were performed to validate the study, whose results will be presented in part II of this work.

The approach followed in this study has general validity and can also be followed to devise the state-space model of a Split-pi operating with a storage-side voltage that is higher than the grid-side voltage or when other bidirectional DC/DC converter topologies are employed to interface an ESS with a DC microgrid. Furthermore, the presented study builds the premises for designing unconventional control systems for the Split-pi that are suitable for operating in more than one microgrid scenario.

Author Contributions: Conceptualization, M.L., A.S., M.P. and M.C.D.P.; methodology, M.L., A.S. and A.A.; software, M.L., A.S., A.A. and G.L.T.; validation, M.L., A.A., M.C.D.P. and M.P.; writingoriginal draft preparation, M.L., A.S. and M.P.; writing-review and editing, M.L., A.A., M.C.D.P. and G.L.T.; supervision, M.P. and M.L.; funding acquisition, M.P. and M.L. All authors have read and agreed to the published version of the manuscript.

Funding: This research was funded by the Italian Ministry of University and Research (MUR), program PON R\&I 2014/2020-Avviso n. 1735 del 13/07/2017-PNR 2015/2020, project "NAUSICA-NAvi efficienti tramite 1'Utilizzo di Soluzioni tecnologiche Innovative e low CArbon," CUP: B45F21000680005.

Institutional Review Board Statement: Not applicable.

Informed Consent Statement: Not applicable.

Data Availability Statement: Not applicable.

Conflicts of Interest: The authors declare no conflict of interest. 


\section{Nomenclature}

\begin{tabular}{|c|c|}
\hline$d$ & Duty cycle \\
\hline $\bar{d}$ & Average duty cycle for state-space model linearization \\
\hline$d_{i}$ & Coefficients of the denominator of the transfer function $G_{p 1}(s)$ \\
\hline $\mathrm{m}_{\varphi}$ & Phase margin \\
\hline $\mathrm{m}_{\mathrm{g}}$ & Gain margin \\
\hline$n_{i}$ & $\begin{array}{l}\text { Coefficients of the numerator of the transfer function } G_{p 1}(s) \text { and the } \\
\text { denominator of } G_{p 2}(s)\end{array}$ \\
\hline$r_{i \%}$ & Current ripple in input/output inductor $L$ \\
\hline$r_{v \%}$ & Voltage ripple in the bulk capacitor $C$ \\
\hline$r_{v e \%}$ & Voltage ripple in the external capacitor $C_{e}$ \\
\hline$u$ & Input vector of the state-space model of the Split-pi converter \\
\hline$x$ & State vector of the state-space model of the Split-pi converter \\
\hline $\bar{x}$ & Average state vector for state-space model linearization \\
\hline$y$ & Output vector of the state-space model of the Split-pi converter \\
\hline & Crossover frequency \\
\hline$A, B, C, D$ & Matrices of the state-space model of the Split-pi converter \\
\hline C & Bulk capacitor of the Split-pi converter \\
\hline$C_{e}$ & External input/output capacitor of the Split-pi converter \\
\hline$C_{e, \min }$ & Minimum capacitance value to obtain the chosen ripple $r_{v e \%}$ \\
\hline$C_{\min }$ & Minimum capacitance value to obtain the chosen ripple $r_{v \%}$ \\
\hline$E_{d}$ & $\begin{array}{l}\text { Equivalent no-load voltage of the aggregated droop-controlled generators of the } \\
\text { microgrid in scenario \#5 after load transformation }\end{array}$ \\
\hline$E_{d s}$ & No-load voltage used to control the storage converter in droop mode \\
\hline$F_{s w}$ & Switching frequency \\
\hline$G_{a d d}(s)$ & $\begin{array}{l}\text { Additional transfer function to be included after the PI regulator of current loop } \\
\text { for } I_{2} \text { in the case of stiff microgrid }\end{array}$ \\
\hline$G_{c i 1}(s)$ & Transfer function of the controller for the internal current loop for $I_{L 1}$ \\
\hline$G_{c i 2}(s)$ & Transfer function of the controller for the external current loop for $I_{2}$ \\
\hline$G_{c v 2}(s)$ & Transfer function of the controller for the voltage loop for $V_{2}$ \\
\hline$G_{p 1}(s)$ & Transfer function of the process that expresses the relationship between $d$ and $I_{L 1}$ \\
\hline$G_{p 2}(s)$ & Transfer function of the process that expresses the relationship between $I_{L 1}$ and $I_{2}$ \\
\hline$I$ & $\begin{array}{l}\text { Current supplied by the aggregated current generators of the microgrid managed } \\
\text { by the EMS }\end{array}$ \\
\hline$I_{1}$ & Input current of the Split-pi converter (port 1, storage-side) \\
\hline$I_{1 n}$ & Nominal input current (storage-side) \\
\hline$I_{2}$ & Output current of the Split-pi converter (port 2, grid-side) \\
\hline$I_{2 n}$ & Nominal output current (grid-side) \\
\hline$I_{2 r e f}$ & $\begin{array}{l}\text { Reference current computed using the EMS for the storage converter controlled } \\
\text { in current mode }\end{array}$ \\
\hline$I_{d}$ & Current supplied by the aggregated droop-controlled generators of the microgrid \\
\hline$I_{c x}$ & Maximum charging current of the storage system \\
\hline$I_{d x}$ & Maximum discharging current of the storage system \\
\hline$I_{e q}$ & $\begin{array}{l}\text { Equivalent current generator considered as active load in scenarios \#1-\#4 after } \\
\text { load transformation }\end{array}$ \\
\hline$I_{L 1}$ & Current of the leftmost inductor of the Split-pi converter (port 1, storage-side) \\
\hline$I_{L 10}$ & Average current value of the leftmost inductor for model linearization \\
\hline$I_{L 2}$ & Current of the rightmost inductor of the Split-pi converter (port 2, grid-side) \\
\hline$I_{L 20}$ & Average current value of the rightmost inductor for model linearization \\
\hline$K_{i i}$ & Integral gain of the PI regulator of current loop for $I_{L 1}$ \\
\hline$K_{i i 2}$ & Integral gain of the PI regulator of current loop for $I_{2}$ \\
\hline$K_{i v}$ & Integral gain of the PI regulator of voltage loop for $V_{2}$ \\
\hline$K_{p i}$ & Proportional gain of the PI regulator of current loop for $I_{L 1}$ \\
\hline
\end{tabular}


$K_{p i 2} \quad$ Proportional gain of the PI regulator of current loop for $I_{2}$

$K_{p v} \quad$ Proportional gain of the PI regulator of voltage loop for $V_{2}$

$L \quad$ Inductor at input/output ports of the Split-pi converter

$L_{\min } \quad$ Minimum inductance value to obtain the chosen ripple $r_{i} \%$

$P_{n} \quad$ Nominal power of the storage converter

$R \quad$ Equivalent load resistance considered in scenarios \#1-\#4 after load transformation

$R_{c} \quad$ Parasitic resistance of bulk capacitor of the Split-pi converter

$R_{d} \quad$ Equivalent droop resistance of the aggregated droop-controlled generators of

$R_{d} \quad$ the microgrid

$R_{d s} \quad$ Droop resistance used to control the storage converter in droop mode

$R_{e} \quad$ Parasitic resistance of external input/output capacitor of the Split-pi converter

$R_{L} \quad$ Parasitic resistance of inductor at input/output ports of the Split-pi converter

$R_{\text {load }} \quad$ Equivalent load resistance of the microgrid

$R_{k} \quad$ Fictitious resistance term appearing in the transfer function $G_{p 2}(s)$

$R_{n} \quad$ Nominal load resistance

SOC State of charge of the storage system

$V_{1} \quad$ Input voltage of the Split-pi converter (port 1, storage-side)

$V_{1 n} \quad$ Nominal input voltage (storage-side)

$V_{2} \quad$ Output voltage of the Split-pi converter (port 2, grid-side)

$V_{2 n} \quad$ Nominal output voltage (grid-side)

$V_{2 \text { ref }}$ Reference output (microgrid) voltage for the storage converter controlled

in droop mode

$V_{c} \quad$ Voltage of the bulk capacitor of the Split-pi converter

$V_{c 0} \quad$ Average voltage value of the bulk capacitor for model linearization

$V_{e} \quad$ Voltage of the external input/output capacitor of the Split-pi converter

$V_{e 0} \quad$ Average voltage value of the external capacitors for model linearization

\section{Appendix A. Coefficients of the Transfer Functions of Model A}

The coefficients of the transfer functions (14) and (15) of state-space model A are expressed using the following equations:

$$
\begin{aligned}
& \left\{\begin{array}{c}
n_{3}=\frac{V_{c 0}+R_{c}\left(I_{L 10}-I_{L 20}\right)}{L} \\
n_{2}=\frac{(1-\bar{d}) I_{L 10}\left(L-C R_{c}^{2}\right)+n_{3} L C\left(R_{p}+R_{L}+R_{c}+\frac{L}{C_{e} R_{s u m}}\right)}{L^{2} C} \\
n_{1}=\frac{(1-\bar{d}) I_{L 10}\left(R_{p}+R_{L}-R_{c}+R_{c} \frac{\frac{L}{R_{c}}-R_{c} C}{C_{e} R_{\text {sum }}}\right)+n_{3} L\left(\frac{C R^{2}}{C_{e} R_{\text {sum }}^{2}}+\frac{C R_{\text {tot }}}{C_{e} R_{\text {sum }}}+1\right)}{n_{0}=\frac{(1-\bar{d}) I_{L 10}\left(R+R_{L}^{2}-R_{c}\right)+n_{3} L}{L^{2} C} \cdot \frac{1}{C_{e} R_{\text {sum }}}}
\end{array}\right. \\
& d_{4}=1 \\
& d_{3}=\frac{R_{p}+2 R_{L}+(2-\bar{d}) R_{c}}{L}+\frac{1}{C_{e} R_{\text {sum }}} \\
& d_{2}=\frac{R+2 R_{L}+(2-\bar{d}) R_{c}}{L} \cdot \frac{1}{C_{e} R_{\text {sum }}}+ \\
& \frac{R_{L}^{2}+R_{L} R_{p}+R_{c}\left((2-\bar{d}) R_{L}+\bar{d}(1-\bar{d}) R_{c}+(1-\bar{d}) R_{p}\right)}{(2-\bar{d}) R_{L}+\bar{d}(1-\bar{d})\left(R_{c}-R_{L}-R_{p}\right)+(1-\bar{d}) R_{p}}+\frac{1+(1-\bar{d})^{2}}{L C} \\
& d_{1}=\frac{(2-\bar{d}) R_{L}+\bar{d}(1-\bar{d})\left(R_{c}-R_{L}-R_{p}\right)+(1-\bar{d}) R_{p}}{L^{2} C}+ \\
& \left(\frac{R_{L}^{2}+R_{L} R+R_{c}\left((2-\bar{d}) R_{L}+\bar{d}(1-\bar{d}) R_{c}+(1-\bar{d}) R\right)}{L^{2}}+\frac{1+(1-\bar{d})^{2}}{L C}\right) \cdot \frac{1}{C_{e} R_{\text {sum }}} \\
& d_{0}=\frac{(2-\bar{d}) R_{L}+\bar{d}(1-\bar{d})\left(R_{c}-R_{L}-R\right)+(1-\bar{d}) R}{L^{2} C} \cdot \frac{1}{C_{e} R_{\text {sum }}} \\
& R_{k}=\frac{(1-\bar{d})\left(V_{c 0}-I_{L 20} R_{c}\right)}{I_{L 10}}-R_{L}
\end{aligned}
$$




\section{Appendix B. Coefficients of the Transfer Functions of Model B}

The coefficients of the transfer functions (24) and (25) of state-space model B are expressed using the following equations:

$$
\begin{gathered}
\left\{\begin{array}{c}
n_{2}=\frac{V_{c 0}+R_{c}\left(I_{L 10}-I_{L 20}\right)}{L} \\
n_{1}=\frac{(1-\bar{d}) I_{L 10}\left(L-C R_{c}^{2}\right)+n_{2} L C\left(R_{L}+R_{c}\right)}{L^{2} C} \\
n_{0}=\frac{(1-\bar{d}) I_{L 10}\left(R_{L}-R_{c}\right)+n_{2} L}{L^{2} C} \\
d_{3}=1 \\
d_{1}=\frac{R_{L}^{2}+R_{c}\left((2-\bar{d}) R_{L}+\bar{d}(1-\bar{d}) R_{c}\right)}{d_{2}=\frac{2 R_{L}+(2-\bar{d}) R_{c}}{L^{2}}+\frac{1+(1-\bar{d})^{2}}{L C}} \\
d_{0}=\frac{(2-\bar{d}) R_{L}+\bar{d}(1-\bar{d})\left(R_{c}-R_{L}\right)}{L^{2} C} \\
R_{k}=\frac{(1-\bar{d})\left(V_{c 0}-I_{L 20} R_{c}\right)}{I_{L 10}}-R_{L}
\end{array}\right.
\end{gathered}
$$

\section{References}

1. Di Piazza, M.C.; Luna, M.; Pucci, M.; La Tona, G.; Accetta, A. Electrical Storage Integration into a DC Nanogrid Testbed for Smart Home Applications. In Proceedings of the 2018 IEEE International Conference on Environment and Electrical Engineering and 2018 IEEE Industrial and Commercial Power Systems Europe (EEEIC / I\&CPS Europe), Palermo, Italy, 12-15 June 2018; pp. 1-5.

2. Chub, A.; Vinnikov, D.; Kosenko, R.; Liivik, E.; Galkin, I. Bidirectional DC-DC Converter for Modular Residential Battery Energy Storage Systems. IEEE Trans. Ind. Electron. 2020, 67, 1944-1955. [CrossRef]

3. Sim, J.; Lee, J.; Choi, H.; Jung, J.H. High Power Density Bidirectional Three-Port DC-DC Converter for Battery Applications in DC Microgrids. In Proceedings of the 2019 10th International Conference on Power Electronics and ECCE Asia (ICPE 2019-ECCE Asia), Busan, Korea, 27-30 May 2019; Volume 3, pp. 843-848.

4. Byrne, R.H.; Nguyen, T.A.; Copp, D.A.; Chalamala, B.R.; Gyuk, I. Energy Management and Optimization Methods for Grid Energy Storage Systems. IEEE Access 2018, 6, 13231-13260. [CrossRef]

5. La Tona, G.; Luna, M.; Di Piazza, A.; Di Piazza, M.C. Towards the real-world deployment of a smart home EMS: A DP implementation on the Raspberry Pi. Appl. Sci. 2019, 9, 2120. [CrossRef]

6. Accetta, A.; Pucci, M. Energy Management System in DC Micro-Grids of Smart Ships: Main Gen-Set Fuel Consumption Minimization and Fault Compensation. IEEE Trans. Ind. Appl. 2019, 55, 3097-3113. [CrossRef]

7. Gorji, S.A.; Sahebi, H.G.; Ektesabi, M.; Rad, A.B. Topologies and Control Schemes of Bidirectional DC-DC Power Converters: An Overview. IEEE Access 2019, 7, 117997-118019. [CrossRef]

8. Beheshtaein, S.; Cuzner, R.M.; Forouzesh, M.; Savaghebi, M.; Guerrero, J.M. DC Microgrid Protection: A Comprehensive Review. IEEE J. Emerg. Sel. Top. Power Electron. 2019, 6777. [CrossRef]

9. Han, S.; Divan, D. Bi-directional DC/DC converters for plug-in hybrid electric vehicle (PHEV) applications. In Proceedings of the 2008 Twenty-Third Annual IEEE Applied Power Electronics Conference and Exposition, Austin, TX, USA, 24-28 February 2008; pp. 784-789.

10. Tytelmaier, K.; Husev, O.; Veligorskyi, O.; Yershov, R. A review of non-isolated bidirectional dc-dc converters for energy storage systems. In Proceedings of the 2016 II International Young Scientists Forum on Applied Physics and Engineering (YSF), Kharkiv, Ukraine, 10-14 October 2016; pp. 22-28.

11. Odo, P. A Comparative Study of Single-phase Non-isolated Bidirectional dc-dc Converters Suitability for Energy Storage Application in a dc Microgrid. In Proceedings of the 2020 IEEE 11th International Symposium on Power Electronics for Distributed Generation Systems (PEDG), Dubrovnik, Croatia, 28 September-1 October 2020; pp. 391-396.

12. Crocker, T.R. Power Converter and Method for Power Conversion. U.S. Patent No. 20040212357A1, 28 October 2004.

13. Khan, S.A.; Pilli, N.K.; Singh, S.K. Hybrid Split Pi converter. In Proceedings of the 2016 IEEE International Conference on Power Electronics, Drives and Energy Systems (PEDES), Trivandrum, India, 14-17 December 2016; pp. 1-6.

14. Sobhan, S.; Bashar, K.L. A novel Split-Pi converter with high step-up ratio. In Proceedings of the 2017 IEEE Region 10 Humanitarian Technology Conference (R10-HTC), Dhaka, Bangladesh, 21-23 December 2017; pp. 255-258.

15. Ahmad, T.; Sobhan, S. Performance analysis of bidirectional split-Pi converter integrated with passive ripple cancelling circuit. In Proceedings of the 2017 International Conference on Electrical, Computer and Communication Engineering (ECCE), Cox's Bazar, Bangladesh, 16-18 February 2017; pp. 433-437.

16. Singhai, M.; Pilli, N.K.; Singh, S.K. Modeling and analysis of split-Pi converter using State space averaging technique. In Proceedings of the 2014 IEEE International Conference on Power Electronics, Drives and Energy Systems (PEDES), Mumbai, India, 16-19 December 2014; pp. 1-6. 
17. Maclaurin, A.; Okou, R.; Barendse, P.; Khan, M.A.; Pillay, P. Control of a flywheel energy storage system for rural applications using a Split-Pi DC-DC converter. In Proceedings of the 2011 IEEE International Electric Machines \& Drives Conference (IEMDC), Niagara Falls, ON, Canada, 15-18 May 2011; pp. 265-270.

18. Sabatta, D.; Meyer, J. Super capacitor management using a Split-Pi symmetrical bi-directional DC-DC power converter with feed-forward gain control. In Proceedings of the 2018 International Conference on the Domestic Use of Energy (DUE), Cape Town, South Africa, 3-5 April 2018; pp. 1-5.

19. Monteiro, V.; Oliveira, C.; Rodrigues, A.; Sousa, T.J.C.; Pedrosa, D.; Machado, L.; Afonso, J.L. A Novel Topology of Multilevel Bidirectional and Symmetrical Split-Pi Converter. In Proceedings of the 2020 IEEE 14th International Conference on Compatibility, Power Electronics and Power Engineering (CPE-POWERENG), Setubal, Portugal, 8-10 July 2020; pp. 511-516.

20. Alzahrani, A.; Shamsi, P.; Ferdowsi, M. Single and interleaved split-pi DC-DC converter. In Proceedings of the 2017 IEEE 6th International Conference on Renewable Energy Research and Applications (ICRERA), San Diego, CA, USA, 5-8 November 2017; pp. 995-1000.

21. Mohan, N.; Undeland, T.M.; Robbins, W.P. Power Electronics. Converters, Applications and Design, 3rd ed.; John Wiley and Sons, Inc.: Hoboken, NJ, USA, 2003.

22. Middlebrook, R.D.; Cuk, S. A general unified approach to modelling switching-converter power stages. In Proceedings of the 1976 IEEE Power Electronics Specialists Conference, Cleveland, OH, USA, 8-10 June 1976; pp. 18-34.

23. Fung, H.-W.; Wang, Q.-G.; Lee, T.-H. PI Tuning in Terms of Gain and Phase Margins. Automatica 1998, 34, 1145-1149. [CrossRef] 